\title{
Analysis of Students' Errors and Misconceptions in Solving Linear Ordinary Differential Equations Using the Method of Laplace Transform
}

\author{
Alfred Mvunyelwa Msomi ${ }^{1,2 *}$ (D), Sarah Bansilal ${ }^{1,2 *}$ (D)
}

${ }^{1}$ Mangosuthu University of Technology, SOUTH AFRICA
${ }^{2}$ University of KwaZulu Natal, SOUTH AFRICA
${ }^{\star}$ Corresponding Author: alfredmsomi@gmail.com

Citation: Msomi, A. M., \& Bansilal, S. (2022). Analysis of Students' Errors and Misconceptions in Solving Linear Ordinary Differential Equations Using the Method of Laplace Transform. International Electronic Journal of Mathematics Education, $17(1)$, em0670. https://doi.org/10.29333/iejme/11474

\section{ARTICLE INFO}

Received: 28 May 2020

Accepted: 3 Aug. 2020

\begin{abstract}
Laplace transform (LT) is an essential mathematical tool for solving linear ordinary differential equations (ODE) with boundary values, by transforming differential equation into algebraic equations which are easier to manipulate. In this article, we analyse the errors students make and misconceptions they have in solving linear ODE using LT method. The study participants were 81 students enrolled in an engineering mathematics course at a University of Technology in South Africa. The students' responses to an item based on LT which formed part of an assessment, were analysed. The analysis identified three stages of working that were necessary to reach a solution (introduction of LT and simplification; resolution of expressions using partial fractions (PF); carrying out the inverse LT and manipulations). Within each stage, we distinguished between three types of errors (conceptual, procedural and technical). The results showed that students experienced most problems when working in the PF layer because of the poor background in manipulation of algebraic expressions. It is recommended that students are given opportunities to develop fluency in pre-requisite concepts, so that their efforts at solving problems using LT or other advanced mathematics techniques can be less stressful.
\end{abstract}

Keywords: engineering mathematics, errors, Laplace transform, misconceptions, ordinary differential equations

\section{INTRODUCTION}

The Laplace transformation (LT) is one of the methods that can be used to solve the nonhomogeneous linear ordinary differential equations (ODE) with the given boundary conditions (Deller, 2009). However, students make numerous errors in the process of solving equations. They do not commit these errors only because of careless mistakes but through intelligent generalisations based on a misunderstanding of underlying concepts (Naseer, 2015). It is difficult to erase errors and misconceptions once embedded in students' memories. (Roselizawati, Sarwadi, \& Shahrill, 2014; Svandova, 2014). Consequently, it is essential that instructors are aware of these common errors and misconceptions so that a solid foundation can be built to prevent and correct misconceptions. Hence, knowledge of errors and misconceptions can be used in order to make informed instructional decisions (Banerjee \& Subramaniam, 2012; Welder, 2012). It is important that instructors are conscious of the correct and incorrect conceptions students have regarding the concept of LT, and potential errors and misconceptions (Almog \& Ilany, 2012). This might not only assist instructors to avoid the creation of such errors and misconceptions, but to remedy those errors and misconceptions (Welder, 2012).

The literature indicates that teachers who consider their students' prior knowledge when planning and preparing classroom activities are able to promote conceptual understanding for students (Banerjee \& Subramaniam, 2012; Brown et al., 2011).

The current study aims to identify the common errors and misconceptions of students from an undergraduate engineering mathematics course through analysis of their written responses to an item based on LT. It is hoped that by sharing these results, other instructors could be better informed about possible areas that could be targeted when teaching the topic.

The analysis of answer scripts of students' errors and misconceptions in solving second order linear ordinary differential equations using LT, in the current study is guided by Sfard's (1992) process-object theory. The model proposes that a mathematics concept can be regarded in two different ways: operationally when a mathematical concept is seen as a process and structurally when a mathematical concept is seen as an object. Being able to see a mathematical concept both as a process and as an object is indispensable for a deep understanding of mathematics (Sfard, 1991). In her study, she viewed a process as a repeated 


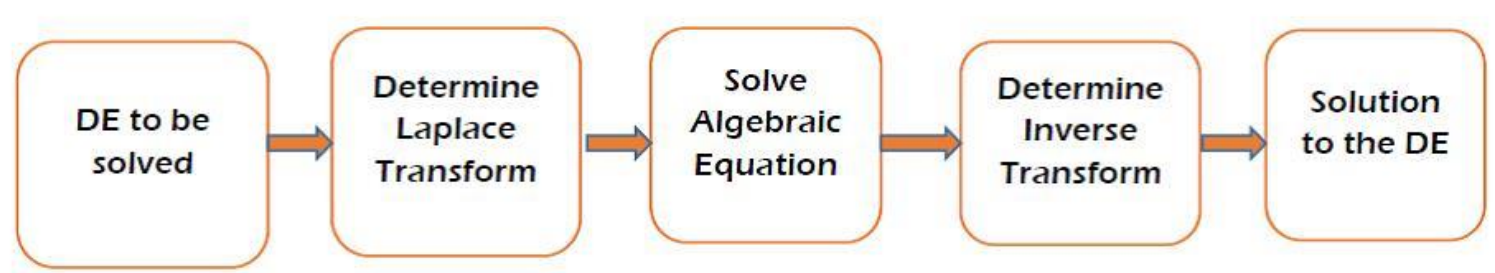

Figure 1. Process of Solution of Differential Equations using Laplace transform

progression of interiorised actions. A process needs to be encapsulated into an object before being acted upon by other processes. Hence an object is viewed as an entity upon which other processes can be carried out. According to Sfard and Linchevski (1994), the structural thinking is associated with an advanced stage of concept development.

Sfard and Linchevski (1994) argue that underlying concepts and principles serve as a connection between prior knowledge and the new knowledge. When the prior knowledge is not well understood, it can impede the development of abstract concepts which are necessary for attaining a structural understanding. Sfard gives three stages that occur during the formation of a concept, that is during the transition from the operational to the structural stages. These three stages are interiorisation, condensation and reification (Sfard, 1991). However, not all students go through these stages; some consequently remain with the pseudo structural conception of the concept. Hence, this current study aims to identify some of the errors and misconceptions that are linked to pseudo-structural conceptions of the concepts in solving differential equations using LT.

A particular contribution of this study is the conceptual flow diagram that has been developed to identify the areas where the error occurred (See Figure 2). This can be used to guide lecturers teaching approaches on the method of LT for the students to minimise the risk of poor performance in this topic.

\section{DEFINITION AND NOTATION OF THE LAPLACE TRANSFORM (LT)}

The LT of a function $f(t)$ is formally defined as

$$
L\{f(t)\}=F(s)=\int_{0}^{\infty} f(t) e^{-s t} d t \text { for } s>0
$$

With $s$ being a complex variable corresponding to time. In the above equation, the exponential function $e^{-s t}$ is defined as the crux of the integral of Laplace and $\int_{0}^{\infty} f(t) e^{-s t} d t$ is known as the Laplace integral of the function $f(t)$. The symbol $L$ is the Laplace transform, which produces a new function $F(s)=L\{f(t)\}$ (Doetsch \& Debnath, 2008).

The steps involved in the process of finding a solution to the linear ordinary differential equation (ODE) are illustrated in Figure 1. The desired solution of differential equation is obtained by taking the inverse transform on the solution of algebraic equation.

\section{LITERATURE REVIEW}

This section summarizes some key findings from pertinent literature exploring students' errors and misconceptions in mathematics learning.

Solution of second order linear ordinary differential equations in mathematics can be obtained by utilising the method of LT. This method converts a given differential equation into an algebraic equation with integration of the boundary conditions from the commencement of the process of finding the solution to the equation (Anumaka, 2012). The LT offers a method of examining a linear system utilising algebraic methods. The fundamental process of analysing a system utilising Laplace transform encompasses transformation of the differential equation into $s$ - domain. The $s$ - domain is considered to transform input functions, discovering an output function through algebraically combing the input and transfer functions as well as utilising partial functions to reduce the output function to simpler components and conversion of output equation back to time-domain (Dass, 2009; Stroud, 2003).

It is important to study students' errors and misconceptions regarding the difficulties involved in learning, and the relevance of the LT in engineering education. This study is part of a larger series of investigations of learning, and the use of physical, technological and symbolic tools for making sense and modelling, in engineering education, especially electrical engineering.

Many mathematics education researchers support the view that errors or misconceptions should be viewed as being part of the process of learning, rather than something that should be eliminated (Kazunga \& Bansilal, 2018; Lannin, Barker, \& Townsend, 2007; Luneta \& Makonye, 2010; Mahlabela \& Bansilal, 2015; Naseer, 2015; Smith, diSessa, \& Roschelle, 1993). Smith et al. (1993) explain that misconceptions develop as part of the process of constructing productive knowledge and learners' conceptions are embedded within complex personal cognitive systems, and are not single units of knowledge.

Misconceptions may be uncovered when students make persistent errors that arise from an underlying misconception, however some errors may be as a result of a careless slip. Hence errors should be taken as "opportunities for deepening one's 
understanding and as important components of learning process" (Lannin, Barker, \& Townsend, 2007, p. 44-45). Errors and misconceptions made by students in the process of solving mathematical problems, differ depending on the particular problem. Roselizawati et al. (2014) concluded that the level of misconceptions seemed to have a significant effect on students' progress and achievement in high stakes tests or examinations. Their study found that students even at the end of their secondary schooling are still struggling with some of the fundamental concepts in mathematics.

Very few studies are found in the literature on errors using the LT method particularly in solving linear ordinary differential second order equations with forcing function forcing that embody periodic functions (Brijlall \& Maharaj, 2017). To better understand the factors leading to students' errors in solving a differential equation using LT, structured studies with various forcing functions on the right hand side of a differential equations should be performed. Current research supports the use of LT in solving differential equations; however, a continuation of current research with various forcing functions on the right hand side of the equation will help identify various errors (Cohen, 2007).

Many studies have pointed out that students find mathematics at university difficult because of the transition from elementary to advanced mathematics itself (Kazunga \& Bansilal, 2020; Tall, 1991). Muzangwa and Chifamba (2012), in their study on the learning of calculus by undergraduate students, found that the students' misconceptions are a result of poor understanding of the basic calculus concepts of limits of functions and their representation. Bezuidenhout (2001) also found that some problems and misconceptions in calculus could be a result of teaching methods which emphasises to a large extent, procedural aspects of the calculus and neglects a solid grounding in the underpinnings of calculus. From the results of the study, by Nurmeidina and Rafidiyah (2019) it was found that students revealed poor conceptual and procedural understanding of integration, and struggled with other topics required for integration, because of a poor background knowledge. Often, one of the reasons for students' poor performance in higher level mathematics is because of a limited background of the prerequisite concepts (Kazunga \& Bansilal, 2020). The authors argue that prerequisite concepts can hinder the learning of a new concept if these were not "encapsulated to the stage where learners can apply them to new situations" (Kazunga \& Bansilal, 2020:6). In their study the authors found that one of the main reasons for students' poor performance on items based on the concept of solving systems of equations was because of their struggles with the prerequisite concepts of determinant and matrix inverse. In this study, the concept of partial fraction (PF) decomposition forms part of the pre-requisite knowledge and is an essential tool for applying LT to ordinary differential equations.

Authors agree that the topic of partial fraction decomposition is a section that many mathematics students find tedious and often struggle with (Brazier \& Boman, 2007; Man, 2012). PF decomposition is not only used in the solution of ordinary differential equations through the LT methods but it is also used in calculus, linear algebra, modern algebra, discrete mathematics and control theory (Brazier \& Boman, 2007; Man, 2012). One approach to working out partial fraction decompositions, is the method of undetermined coefficients where the unknowns are solved by a system of linear equations, which was the approach followed by the participants in this course. Recent research has focused on different approaches to PF decomposition, such as the Heaviside cover -up technique (Brazier \& Boman, 2007; Man, 2007, 2009, 2012).

\section{METHODOLOGY}

The study utilised a qualitative research approach so as to understand the errors and misconceptions identified in the students' responses on question of LT from their examination scripts. The underlying research question guiding this study is: What is the nature of the errors made by students when working with a Laplace transform problem? Ethical issues stipulated by the university rules were taken into consideration and furthermore students' names from the examination scripts were withheld and only codes were used. The sample for this study included 80 students enrolled in a University of Technology mathematics course offered to the Electrical Engineering students. Students ages were between 17 and 21 years old.

\section{Data Collection and Instruments}

The data was collected from the students' responses to a summative mathematics assessment. The analysis of the written responses focussed on errors and misconceptions made by students in solving the following differential equation:

$$
q^{\prime \prime}(t)+q^{\prime}(t)+5 q(t)=17 \sin 2 t
$$

given that $y(0)=0$ and $y^{\prime}(0)=0$

\section{Data Analysis Procedures}

In investigating the errors and misconceptions in the answer scripts, only incorrect and partially correct answers were considered. The framework in Figure 2 was developed to guide the analysis of the errors and misconceptions made by the students. Using this framework, three stages of the solution of second order linear ordinary differential equations using the method of LT, was distinguished:

Stage 1: Introduction of LT on both sides of the equation and simplification

Stage 2: Partial fraction expansion

Stage 3: Inverse LT and solution of differential equations.

A further layer of analysis was then done by following the work of Kiat (2005) to categorise three types of errors that could occur at each of the three stages and noting the frequencies in each of the three stages. These errors were: 


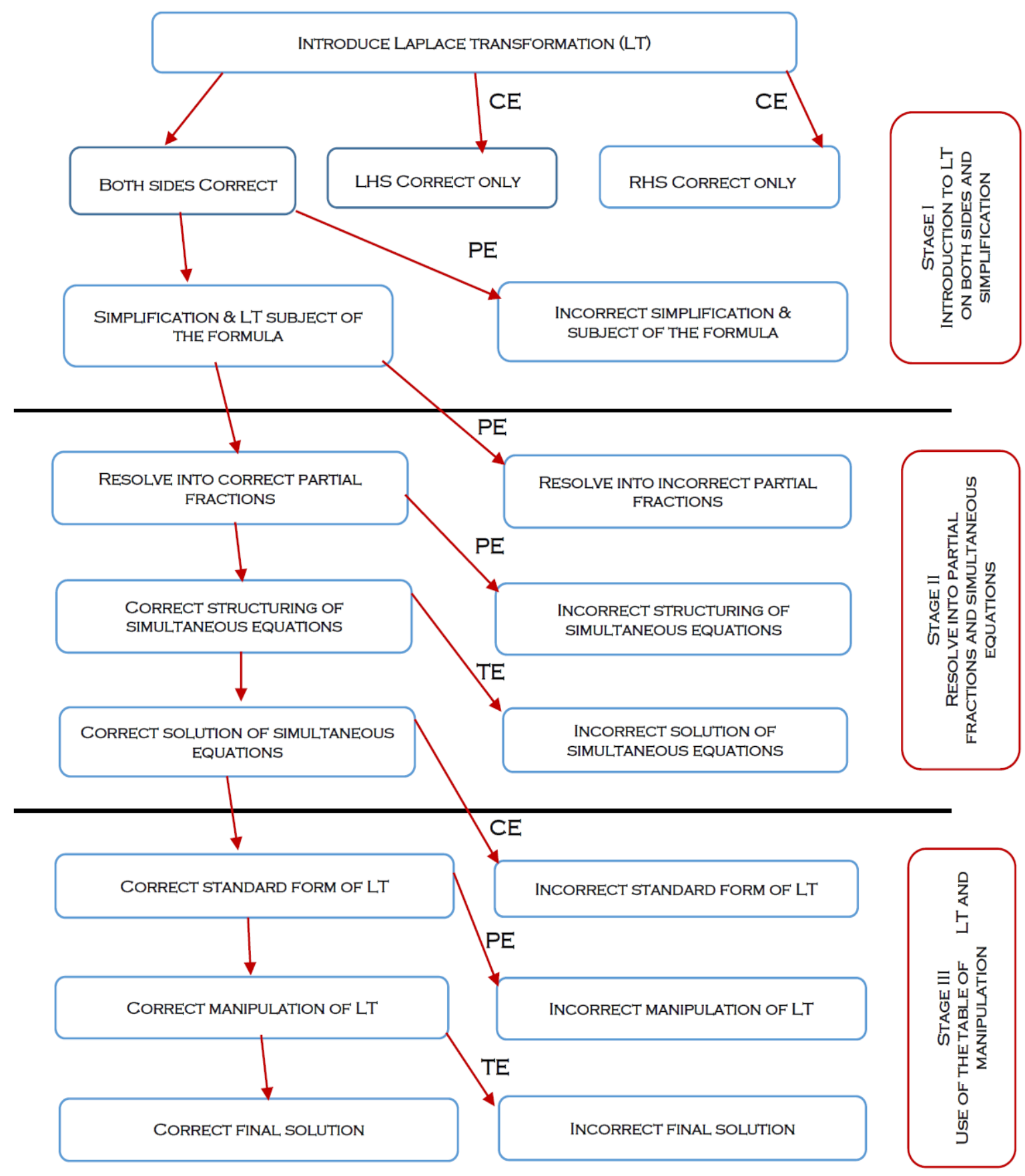

Figure 2. Flow diagram indicating students' errors

Conceptual: These errors are related to a misunderstanding and interpretation of concept. Following the definition by Arslan, conceptual errors are defined as those that are made because of not achieving learning that involves understanding and interpreting concepts and the relations between concepts (Arslan, 2010). An example of a conceptual error is when students do not recognise the LT that should be introduced on both sides of the differential equation when asked to solve a problem using the LT.

Procedural: Procedural errors are those which arise while trying to carry out a procedure, having understood the main concepts behind the problem. In this study procedural errors refer to lack of skills in executing the procedures flexibly, appropriately, and accurately. For example, not being able to carry out the simplification or changing the subject of the formula.

Technical: Godden et al (2013) explains that these errors are a result of slips or silly mistakes that students display. For example, a technical error could be one that occurs while manipulating simultaneous equations. Accordingly, the frequencies of the sub-types of technical errors were calculated only for the related stages in which such errors may occur (see both Table 3 and 4).

Figure 2 illustrates the three stages of the solution shown as a flow diagram where the three types of errors are indicated as CE (Conceptual Errors), PE (Procedural Errors) and TE (Technical Errors) for each of the three stages. 


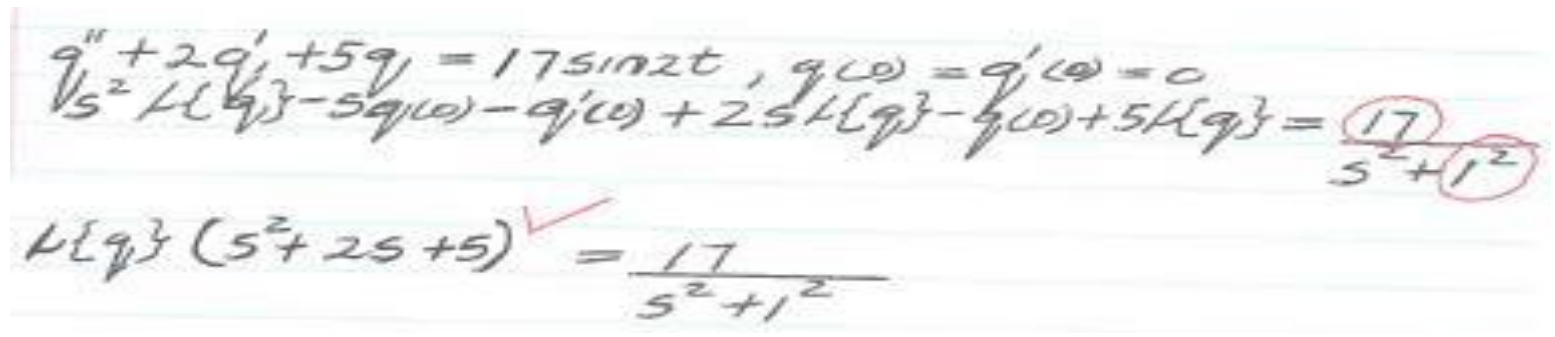

Figure 3. Conceptual error on the right hand side of the equation (Stage I)

Table 1. Results at Stage I (Introduction of Laplace transform on both side and simplification)

\begin{tabular}{cccccc}
\hline Number of students & Conceptual errors & Procedural errors & Wrong Method (did not use LT) & NEC & NAQ \\
\hline 80 & 5 & 3 & 34 & 37 \\
\hline$\%$ & $\mathbf{6}$ & $\mathbf{4}$ & $\mathbf{4 3}$ & $\mathbf{1}$ \\
\hline
\end{tabular}

Table 2. Results at Stage II (Partial fraction expansion and simultaneous equations)

\begin{tabular}{ccccc}
\hline Number of students & Procedural errors & Technical errors & \\
\hline 37 & 5 & 15 & \\
\hline$\%$ & $\mathbf{1 4}$ & $\mathbf{4 1}$ & \\
\hline
\end{tabular}

\section{RESULTS}

The results are presented in terms of each of the three stages.

\section{Stage I}

Stage I of the solution involved the introduction of LT on both sides of the equation and the simplification. An example of a conceptual error ocurring at the first stage of the students solution is given in Figure 3.

As shown in Figure 3, the students experienced challenges in expressing the forcing function (right hand side of the equation) using the given table of LT. A common misconception was the use of an incorrect definition for the LT of a given function. The conceptual error indicated in Figure 3 is the student's use of $s^{2}+1^{2}$ instead of $s^{2}+2^{2}$, since the coefficient of $t$ in the forcing function is 2 but not 1 . The correct formula in this case is: $\sin a t=\frac{a}{s^{2}+a^{2}}$. In general, if the sine wave function is multiplied by a constant namely $k$, then $k \sin a t=\frac{k a}{s^{2}+a^{2}}$. An instructional suggestion would be to emphasise the correct identification of the formula to be used based on the given forcing function and substitution. Perhaps these conceptual errors can be reduced carefully selecting and presenting examples of such errors to students allowing them to challenge their own assumptions.

Table 1 presents the results for errors made by students at the first stage (introducing Laplace transform), where NEC represents no error committed and NAQ represents not attempted question. Note that all 80 responses were analysed for errors made during the first stage.

Table 1 shows that there were 34 students who tried to use a different method called undetermined coefficients instead of LT although the instruction specified the use of the LT. This method gives the same answer to the linear differential equations. From the analysis, it was found that even if students changed to undetermined coefficients ' methods, they did not make it to the final stage and get correct answer. It seems as if some of them deliberately chose the undetermined coefficients method, because they wanted to avoid the method of LT while others may not have understood the difference between the two methods. Table 1 shows that there were 5 students who displayed conceptual errors while only 3 made procedural errors. Considering only those students who followed the instruction and opted for the LT method, it is noteworthy that $87 \%$ passed through the first stage without making any errors. This shows that Stage I was not the major reason for students' poor performance in the item.

\section{Stage II}

Recall that Stage II, is the PF expansion and solution of the associated simultanoeous equations. The analytic framework in Table 2 identifies possible procedural and technical errors in this stage. Procedural errors occur when resolving a fraction into incorrect partial fractions because of a misunderstanding of the rules. Procedural errors are also identified when there is an incorrect structuring of system of equations. Errors that occur when obtaining an incorrect solution of the system of equations were taken as technical errors.

PF decomposition is an important topic in differential equations and some areas of pure or applied mathematics. One familiar method to work out the PF is to employ undetermined coefficients method. An alternative method is to use the Heaviside's coverup technique, that utilises substitutions to establish the unknown coefficients of the PF with single poles, and sequential differentiations to deal with several poles (Norman, 1990). However, the calculations involved might be frustrating for the latter case. 


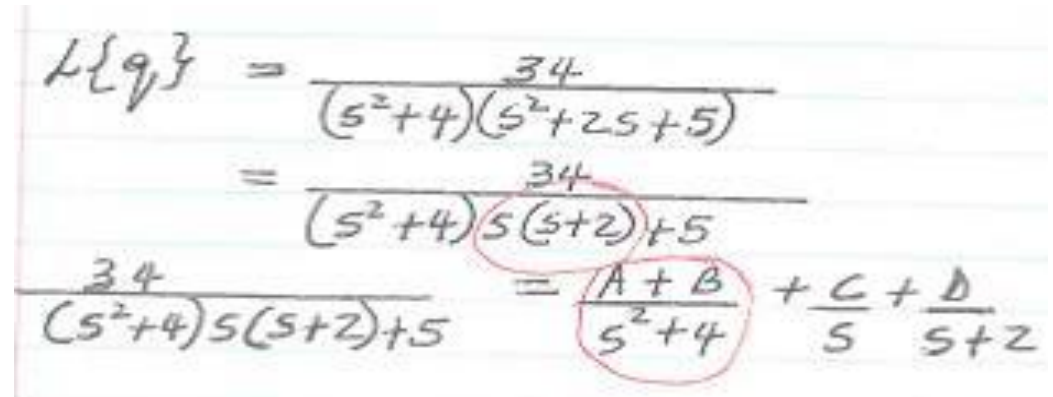

Figure 4. Incorrect resolution into partial fraction

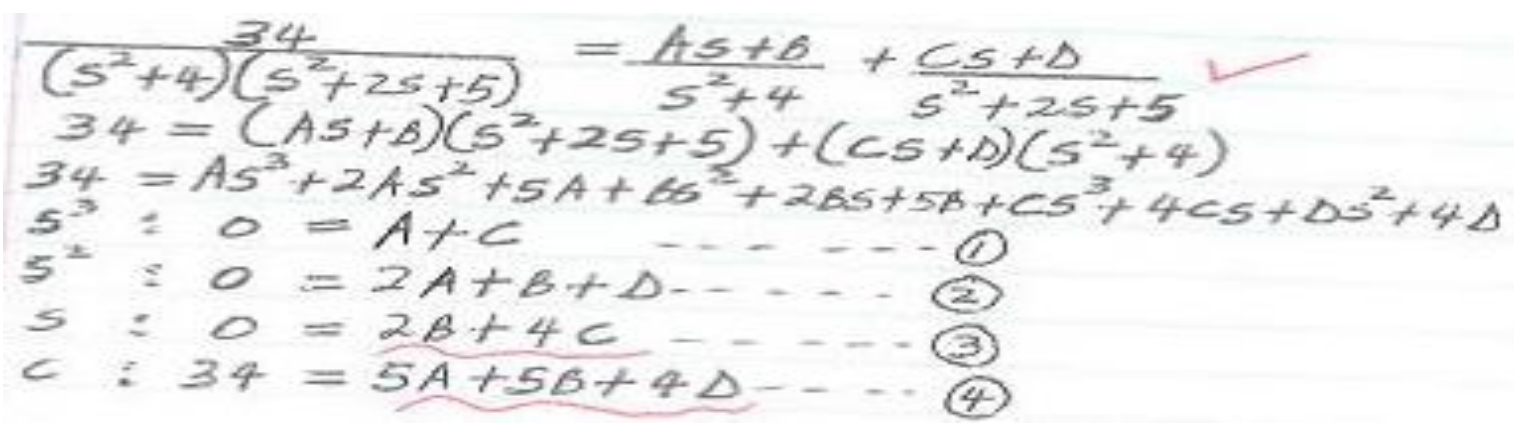

Figure 5. Procedural Error and misconceptions (Stage II)

Students come across PF expansions in first-year calculus for the first time and find it to be useful when working with rational functions. This is relevant to Johann Bernoulli's claim in 1702 that "such a factoring was always possible, and therefore all rational functions could be integrated" (Paor, 2003: 440). Bernoulli's concept began the pathway to Liouville's "Integration in finite terms" (Man, 2007). Students also see PF in differential equations to assist in calculating inverse LT, and in linear algebra using linear systems.

Partial fraction decomposition is also a topic that is commonly taught to school and university students with applications in differential calculus, differential equations and other mathematics subject areas (Kim \& Lee, 2016). Hypothetically, every rational function has a distinct PF decomposition but decomposing a rational function into PF can be an intensive exercise. PF decomposition was one of the challenges students experienced in solving second order linear ODE as indicated by Figure 4.

As shown in Figure 4, students factorised $s^{2}+2 s+5$ to be $s(s+2)+5$ instead of $(s+1)^{2}+4$ by the method of completing the square which is a procedural error. Factorisation by completing the square are taught at school level, and perhaps this misconception may be because of an over dependence on the use of the calculators. This was coded as a procedural error because of the incorrect PF resolution.

In solving a LT problem, some manipulations must be done before $L\{q\}$ can be inverted since it does not appear directly in the table of LT. The method of PF is a technique for decomposing functions like $L\{q\}$ above so that the inverse transform can be determined in a straightforward manner. This technique uses PF expansion to split up complex fraction into forms that are in the table of LT. The correct PF expansion is:

$$
\frac{34}{\left(s^{2}+4\right)\left(s^{2}+2 s+5\right)}=\frac{A s+B}{s^{2}+4}+\frac{C s+D}{s^{2}+2 s+5}
$$

The PF expansion given above, involves two irreducible quadratic factors requiring students to have appropriate cognitive approach because it is a condition that involves four constants. There is a need for students to think logically about the structure of partial fractions determined by certain rules of expansion and apply the rules of PF expansion.

Figure 5 indicates a procedural error where the student struggled to develop the simultaneous equation from the PF that s/he had correctly expanded.

The equations 3 and 4 in Figure 5 are incorrect, as the system of equations was supposed to be given by:

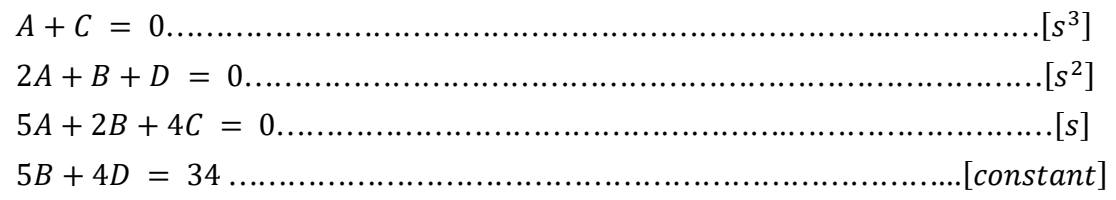

The student's error is classified as procedural according to the analytic framework because of an incorrect structuring of the simultaneous equations when trying to equate the coefficients of the cubic equation on both sides. Hence, the values of the constants will be incorrect because the wrong system of equations was generated. Appropriate procedural fluency has an essential role in dealing with systems of equations; students are expected to apply knowledge of procedures to achieve the correct results. It is a concern that the misconception displayed was that of multiplication of terms with variables, a concept which is encountered 


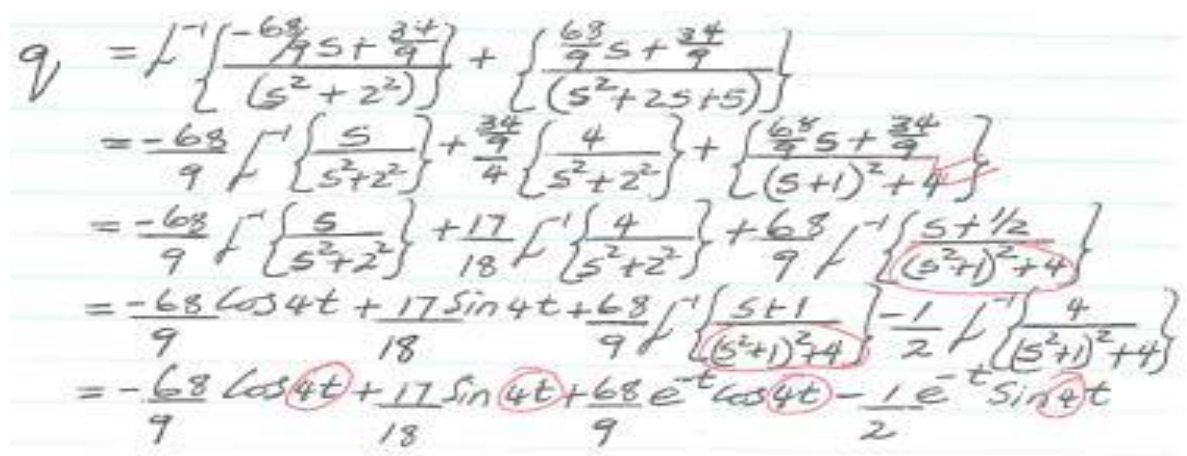

Figure 6. Example of procedural error on inverse Laplace transform (Stage III)

Table 3. Results at Stage III (Inverse Laplace transform and solution of differential equations)

\begin{tabular}{ccccc}
\hline Number of students & Conceptual errors & Procedural errors & \multicolumn{2}{c}{ Technical errors } \\
\hline 17 & 0 & 7 & 4 & NEC \\
\hline$\%$ & 0 & $\mathbf{4 1}$ & $\mathbf{2 3 . 5}$ & \\
\hline
\end{tabular}

in early algebra, Welder (2012) noted that the notational use of brackets should be encouraged at all levels of instruction. Perhaps these algebraic misconceptions may be limited by helping students engage with the underlying structure of simultaneous equations before solving higher order equations.

Table 2 presents a summary of the errors identified at the stage of PF expansion and solving simultaneous equations. Note that the analysis in Table 2 considers the 37 students only from Table 1 who did not make any errors at Stage I. The data analysis of the students' answer scripts shows that $14 \%$ committed procedural errors, whilst $41 \%$ made technical errors and $46 \%$ did not have any errors.

\section{Stage III}

The last step in the process of solving the problem in an ODE is to find the inverse transform on the solution of an algebraic equation in the s-domain using the transformation. The inverse LT takes us to the desired solution in the time domain. Partial fraction expansion, completing the square on second order complex roots of the polynomials and other processes such as time shifting plays an important role in finding the solution. For certain problems, there is not much left to do but to read directly from the table for the correct functions.

Students had difficulty in manually completing the square of this polynomial in order to identify the appropriate formula from the LT table. While the inverse LT process can be studied at a meaningful mathematical level, stage III is concerned with the approach of using PF expansion approach so as to identify the inverse LT from the table of LT. Nonetheless, this approach is not simple and algebraic manipulations are usually required before an inverse LT can be found. Figure 6 illustrates a procedural error at this stage where the student incorrectly expressed the irreducible quadratic expression $s^{2}+2 s+5$ as $\left(s^{2}+1\right)^{2}+4$.

Through analysis of the students' incorrect answers in this study, it became apparent that most of the misconceptions with decomposition of irreducible quadratic expression were rooted in their misconception with completing the square and use of the formula.

Naseer (2015) and Welder (2012), indicated that students come to class with beliefs, ideas, and mathematical concepts that they have developed over the years in their previous classrooms, and life experiences contributing to what and how they learn. Therefore, in developing a solid foundation and preventing and correcting misconceptions, it is essential that the instructors distinguish these common errors and misconceptions. The errors and misconceptions can be utilised to advise instructional decisions and consequently design instructions.

When the system of equations and the unknown constants are correct, $q$ should be expressed by:

$$
q=L^{-1}\left\{\frac{-4 s+2}{s^{2}+4}\right\}+L^{-1}\left\{\frac{4 s+6}{s^{2}+2 s+5}\right\}
$$

The first term on the right hand side can be split into two fractions and then the table of LT can be used to get the desired solution. Some students struggled to get an answer because they could not identify the correct and relevant property from the table of LT which resulted in conceptual errors.

The last term on the right hand side of the equation consists of the polynomial $s^{2}+2 s+5$ which has complex roots and is therefore irreducible. When faced with this irreducible polynomial, students struggled to manipulate the expression using the completion of the square technique in order to apply appropriate formula in their LT table. Table 3 presents a summary of the different errors that were identified at Stage III, noting that the analysis at this stage considers only the 17 students who did not make any error at Stage II in Table 2.

It can be noted from Table 3 that $41 \%$ of students committed procedural errors while $24 \%$ made technical errors. It is important to note that $35 \%$ of students who reached this stage did not commit errors. This observation implies that students' problems are not only in Stage III, but errors at all the stages contributed to the students' poor performance. 


\section{DISCUSSION AND CONCLUSION}

The current study is concerned with errors students make in solving a differential equation using the method of LT. Overall, the study shown that the engineering mathematics students make numerous errors in solving linear ordinary differential equations with constant coefficients using the method of the LT. Lack of understanding of LT concept lead to weak interpretation that has led to conceptual, procedural and technical errors which eventually contribute to students' poor performance. The results of this paper showed that students tend to consider routine learning and hence they learn by rote rules of LT without attempting to master the concept (Skemp, 1987). Perhaps emphasis on relational and conceptual understanding in teaching the concept of LT may result in improved results.

The study followed the students' responses through three stages (Introduction to Laplace transform, partial fraction, inverse Laplace transform) and found that the students encountered problems, at all three stages of the problem. However, a detailed analysis of the errors at the three stages (Introduction of Laplace transform, partial fraction, inverse Laplace transform), showed that the students had most success at the initial stage of identifying the Laplace transform where $87 \%$ of the students who attempted the LT approach did not make any errors. Of the students that proceeded to the second stage, 17 (46\%) were successful at this stage, and 20(54\%) were not. In considering the 17 who reached the third stage of applying the inverse LT, 6 (35\%) were successful. Hence it can be seen that the area of simplifying using PF and simultaneous equations formed the biggest hurdle for these students. The PF expansion and simultaneous equations was considered as a second stage in the analysis of students' errors in this study, and has shown that students will keep on making procedural errors if the prerequisite concepts of PF, decomposition of quadratic expression as well as solving simultaneous equations have not been understood. Kazunga and Bansilal (2020) investigated the role of the prerequisite concepts of determinant and inverse matrix when working with problems on solving systems of equations. The authors showed that the participants in their study carried forward their errors in the prerequisite concepts into their methods of solving the systems of equations. The authors argue that if a prerequisite concept was not understood fully, then further developments that rely on that prerequisite concept become compromised and more complicated. This observation is supported in this study which found that the limited procedural fluency in the prerequisite concepts (PF; decomposition of quadratic expression; solving simultaneous equations) represented the biggest challenges in applying the LT to solve the problem. Fisher and Frey (2012) noted that error analysis can assist in targeting exact misunderstandings instead of reteaching the entire concept. However, it is important to note that simply teaching the formula of PF expansion and the steps to solve simultaneous equations is not sufficient to assist students' improve conceptual understanding (Sweetland \& Fogarty, 2008). Instructors need to explore different methods to approach the PF decomposition that students may find easier to work with.

For some students, the sources of the problems encountered at Stage III of the LT problem was because they did not understand or may not have been aware of how use the table. The correct use of the table demonstrates understanding of inverse LT and solution of differential equations (Fisher \& Frey, 2012).

Our findings provide evidence for previous studies which indicate that students' errors are not deliberate, instead, they are frequently based on procedures used systematically (Cox, 1975). This study found that students' poor knowledge of school level mathematics such as factorisation of a quadratic expression or decomposition of an irreducible quadratic led to them making errors in this problem. Understanding student's thinking in solving a mathematical problem contribute immensely in understanding their underlying challenges (Ching et al., 2016; Hunt \& Little, 2019). Hence there is a need for instructors to learn more about the underlying challenges experienced by students. As the procedural errors can assist instructors to understand the students' difficulties and appraise the knowledge level among students, it is essential that the instructor must be aware of students' pre-conceptions. A simple intervention in the form of revising the topic of quadratic expressions, may help the students in solving the LT problems more effectively.

It is important that instructors teaching the method of LT are more tolerant of students who struggle with LT and endeavour to understand the students' errors and underlying problems. More efforts must be made when introducing the topic so that the links to the other concepts are highlighted so that students have a greater chance of understanding of the concepts immediately after the teaching, which will reduce the amount of errors made later. Thorough practice and coaching at the introduction stage of the concept, the depth of understanding can be increased and improve results. The students should be given a clear idea about every step involved in the process of solving differential equations using the method of LT and every mathematical operation involved should be conceptually grounded.

Our study joins other studies (such as Brijlall \& Maharaj, 2017) on exploring students' understanding of LT method in solving linear ordinary differential equations. This study has shown that many of the procedural and technical errors made by students are related to a poor understanding of mathematics studied in previous levels of study. Although some errors committed by students were because of unfamiliarity with the table of LT, they could not, however choose the correct formula using the standard LT table.

The findings provide evidence for previous studies which indicate that students' errors are not deliberate, instead, they are frequently based on procedures used systematically.

\section{REFERENCES}

Almog, N., \& Ilany, B. S. (2012). Absolute value inequalities: High school students' solutions and misconceptions. Educational Studies in Mathematics, 81(3), 347-364. https://doi.org/10.1007/s10649-012-9404-z 
Banerjee, R., \& Subramaniam, K. (2012). Evolution of a teaching approach for beginning algebra. Educational Studies in Mathematics, 80(3), 351-367. https://doi.org/10.1007/s10649-011-9353-y

Bezuidenhout, J. (2001). Limits and continuity: Some conceptions of first-year students. International Journal of Mathematical Education in Science and Technology, 32(4), 487-500. https://doi.org/10.1080/00207390010022590

Brijlall, D., \& Maharaj, A. (2017). Using groupwork as a learning strategy of Laplace Transform by engineering students. PONTE International Journal of Sciences and Research, 73(6), 345-354. https://doi.org/10.21506/j.ponte.2017.6.29

Brown, I. A., Davis, T. J., \& Kulm, G. (2011). Pre-service teachers' knowledge for teaching algebra for equity in the middle grades: A preliminary report. Journal of Negro Education, 80(3), 266-283.

Ching, Y. H., Yang, D., Baek, Y. K., \& Baldwin, S. (2016). Enhancing graduate students' reflection in e-portfolios using the TPACK framework. Australasian Journal of Educational Technology, 32(5), 108-122. https://doi.org/10.14742/ajet.2830

Cox, L. S. (1975). Systematic Errors in the Four Vertical Algorithms in Normal and Handicapped Populations. Journal for Research in Mathematics Education, 6(4), 202. https://doi.org/10.2307/748696

Deller, J. R. (2009). Laplace transformation. In Fundamentals of Circuits and Filters (pp. 3-1-3-45). https://doi.org/10.3139/9783446461819.005

Doetsch, G., \& Debnath, L. (2008). Introduction to the Theory and Application of the Laplace Transformation. IEEE Transactions on Systems, Man, and Cybernetics, 7(8). https://doi.org/10.1109/tsmc.1977.4309792

Fisher, D., \& Frey, N. (2012). Making time for feedback. In Educational Leadership, 70(1), 42-47.

Godden, H., Mbekwa, M., \& Julie, C. (2013). an Analysis of Errors and Misconceptions in the 2010 Grade 12 Mathematics Examination: a Focus on Quadratic Equations and Inequalities. Proceedings of the 19th Annual Congress of the Association for Mathematics Education of South Africa, 1, 70-79.

Hunt, J. H., \& Little, M. E. (2019). Intensifying Interventions for Students by Identifying and Remediating Conceptual Understandings in Mathematics. Teaching Exceptional Children. https://doi.org/10.1177/0040059914534617

Kazunga, C., \& Bansilal, S. (2018). Misconceptions about determinants. In S. Stewart, C. Andrews-Larson, A. Berman, \& M. Zandieh (eds.), Challenges and Strategies in teaching Linear Algebra pp. 127-145, Springer: Dordrecht. https://doi.org/10.1007/978-3319-66811-6_6

Kazunga, C., \& Bansilal, S. (2020). An APOS analysis of the understanding of solving systems of equations using the inverse matrix method. Educational Studies in Mathematics, 103, 339-358. https://doi.org/10.1007/s10649-020-09935-6

Kim, Y., \& Lee, B. (2016). Partial Fraction Decomposition by Repeated Synthetic Division. American Journal of Computational Mathematics, 6(2), 153-158. https://doi.org/10.4236/ajcm.2016.62016

Lannin, J. K., Barker, D., \& Townsend, B. E. (2007). How students view the general nature of their errors. Educational Studies in Mathematics, 66(1), 43-59. https://doi.org/10.1007/s10649-006-9067-8

Mahlabela, P. T., \& Bansilal, S. (2015). Using theorems-in-action to understand learners' strategies in problem solving in ratio and proportion. Pythagoras, 36(2), 11-20 Art. \#252. https://doi.org/10.4102/pythagoras.v36i2.252

Man, Y. K. (2007). A simple algorithm for computing partial fraction expansions with multiple poles. International Journal of Mathematical Education in Science and Technology, 38(2), 247-251. https://doi.org/10.1080/00207390500432337

Man, Y. K. (2009). An improved Heaviside approach to partial fraction expansion and its applications. International Journal of Mathematical Education in Science and Technology, 40(6), 808-814. https://doi.org/10.1080/00207390902825310

Man, Y. K. (2012). Introducing the improved Heaviside approach to partial fraction decomposition to undergraduate students: Results and implications from a pilot study. International Journal of Mathematical Education in Science and Technology, 43(7), 911-922. https://doi.org/10.1080/0020739X.2012.662292

Muzangwa, J., \& Chifamba, P. (2012). Analysis of Errors and Misconceptions in the Learning of Calculus by Undergraduate Students. Acta Didactica Napocensia, 5(2), 1-10.

Naseer, M. S. (2015). Analysis of Students' Errors and Misconceptions in pre-University Mathematics Courses. In M. N. Salleh, \& N. F. Z. Abedin, (Eds.), Proceedings: First International Conference on Teaching \& Learning 2015 (p. 34-39). Langkawi, Malaysia: MNNF Publisher. ISBN 978-967-13637-1-3.

Nurmeidina, R., \& Rafidiyah, D. (2019). Analysis of Students' Difficulties in Solving Trigonometry Problems. The Mathematics Educator. https://doi.org/10.4108/eai.7-8-2019.2288422

Roselizawati, H. J., Sarwadi, H., \& Shahrill, M. (2014). Understanding Students' Mathematical Errors and Misconceptions: The Case of Year 11 Repeating Students. Mathematics Education Trends and Research. https://doi.org/10.5899/2014/metr-00051

Sfard, A. (1991). On the dual nature of Mathematical conceptions: Refl ections on processes and objects as different sides of the same coin. Educational Studies in Mathematics, 22, 1-36. https://doi.org/10.1007/BF00302715

Sfard, A., \& Linchevski, L. (1994). The gains and the pifalls of reification-the case of algebra. Educational Studies in Mathematics, 26, 191-228. https://doi.org/10.1007/BF01273663

Smith, J. P., diSessa, A. A., \& Roschelle, J. (1993). Misconceptions reconceived: A constructivist analysis of knowledge in transition. The Journal of the Learning Science, 3(2), 115-163. https://doi.org/10.1207/s15327809jls0302_1

Svandova, K. (2014). Secondary school students' misconceptions about photosynthesis and plant respiration: Preliminary results. Eurasia Journal of Mathematics, Science and Technology Education, 10(1), 59-67. https://doi.org/10.12973/eurasia.2014.1018a 
Sweetland, J., \& Fogarty, M. (2008). Prove It! Engaging Teachers as Learners to Enhance Conceptual Understanding. Teaching Children Mathematics, 15(2), 68-73.

Welder, R. M. (2012). Improving Algebra Preparation: Implications from Research on Student Misconceptions and Difficulties. School Science and Mathematics, 112(4), 255-264. https://doi.org/10.1111/j.1949-8594.2012.00136.x 